\begin{tabular}{|l|l|l|l|l|}
\hline Cuadernos I. Geográfica & 24 & pp. 69-87 & Logroño & 1998 \\
\hline
\end{tabular}

\title{
VALORACIÓN DE LOS RECURSOS PASTORALES DE CAMPOS ABANDONADOS DE CAMERO VIEJO (SISTEMA IBÉRICO RIOJANO).
}

\author{
E. E. RODRIGUEZ MERINO ${ }^{1}$ \\ M. P. ERREA ABAD ${ }^{2}$ \\ T. LASANTA MARTINEZ $Z^{2}$
}

\begin{abstract}
RESUMEN: Se calcula la producción pascícola y la capacidad potencial de carga ganadera que admiten los campos abandonados de Camero Viejo. A partir del método del "Valor Pastoral" se obtiene la producción en Unidades Forrajeras (U.F.) de parcelas colonizadas por Genista scorpius y por Cistus laurifolius, como especies más características de los modelos dominantes de sucesión vegetal. Se discute sobre la posible recuperación ganadera de los mejores campos abandonados.

ABSTRACT: The pasture production and the potential capacity of livestock charge that abandoned fields of Camero Viejo can support are calculated. Using the "Pastoral Value" method we can know the production measured in Fodder Units (U.F.) from plots colonized by Genista scorpius and Cistus laurifolius, as the most characteristic species in the dominant models of vegetal succession. The possibilities that the most productivite abandoned fields could be recuperated as grazing lands is also discussed.
\end{abstract}

Palabras clave: Campos abandonados, ganadería extensiva, pastos, montaña, Sistema Ibérico.

Key words: Abandoned fields, livestock, pastures, mountain areas, Iberiam System 
Desde los años setenta la ganadería extensiva constituye la base económica de las pocas explotaciones activas que hay en Camero Viejo (valles del Leza y Jubera). Esta ganadería basa su fuente de alimentación en el aprovechamiento de pastos naturales, con un complemento de pienso (cebada, alfalfa y paja fundamentalmente), que los ganaderos compran en municipios del llano riojano, dada la escasa superficie de cultivos y prados en Camero Viejo.

Los campos abandonados, por la elevada extensión que ocupan y por poseer los suelos más fértiles, constituyen el principal recurso pastoral. No obstante, su productividad pascícola presenta una alta heterogeneidad en relación con el sustrato litológico, la topografía, la fertilidad edáfica, la edad de abandono, la distancia a los establos y la gestión que han soportado y soportan.

En este trabajo se estudia la producción de varios campos abandonados a lo largo del año, representativos de los que actualmente se pastan. Se trata, por un lado, de evaluar su valor pastoral y la carga ganadera que pueden soportar en la actualidad y, por otro lado, de calcular de forma aproximada, qué supondría la incorporación, mediante desbroce selectivo, al sistema pastoral de campos abandonados cubiertos totalmente de matorral. En este sentido, conviene señalar que desde el año 1989 se asiste al desbroce de algunas laderas de matorral y de campos abandonados en diferentes municipios de la Sierra. El objetivo perseguido no es exclusivamente pastoral, sino también controlar la propagación de posibles incendios.

Los estudios de sucesión vegetal en campos abandonados cuentan ya con cierta tradición en la bibliografía española (ver los trabajos de Llorente y Luengo, 1986; Pérez Chacón y Vabre, 1986, Zuazúa, 1987; Zuazúa et al., 1988, Francis, 1990, Montserrat, 1990, entre otros). En Camero Viejo también existe información al respecto (Fernández González, 1980 y 1985; García-Ruiz, et al., 1985; Sobrón y Ortiz, 1989), si bien ninguno de los estudios precedentes realiza una aproximación cuantitativa del potencial pascícola de campos abandonados. En este sentido, destaca el reciente informe realizado por Rodríguez y Errea (1997) para el Instituto de Estudios Riojanos (Gobierno de La Rioja), del que este trabajo toma parte de la información, siguiendo una línea de investigación aplicada en otras áreas: Prepirineo Aragonés (Ascaso, 1990), Alto Maestrazgo (Ferrer et al., 1993), Depresión Central del Ebro (Errea, 1995) y Altos Valles Pirenaicos (Molinillo et al., 1994 y 1997), por poner algunos ejemplos de zonas relativamente próximas.

\section{Area de estudio}

Los ríos Leza y Jubera drenan la comarca riojana de Camero Viejo, con una extensión aproximada de $329 \mathrm{Km}^{2}$ (Fig.1). Constituye una zona montañosa de 


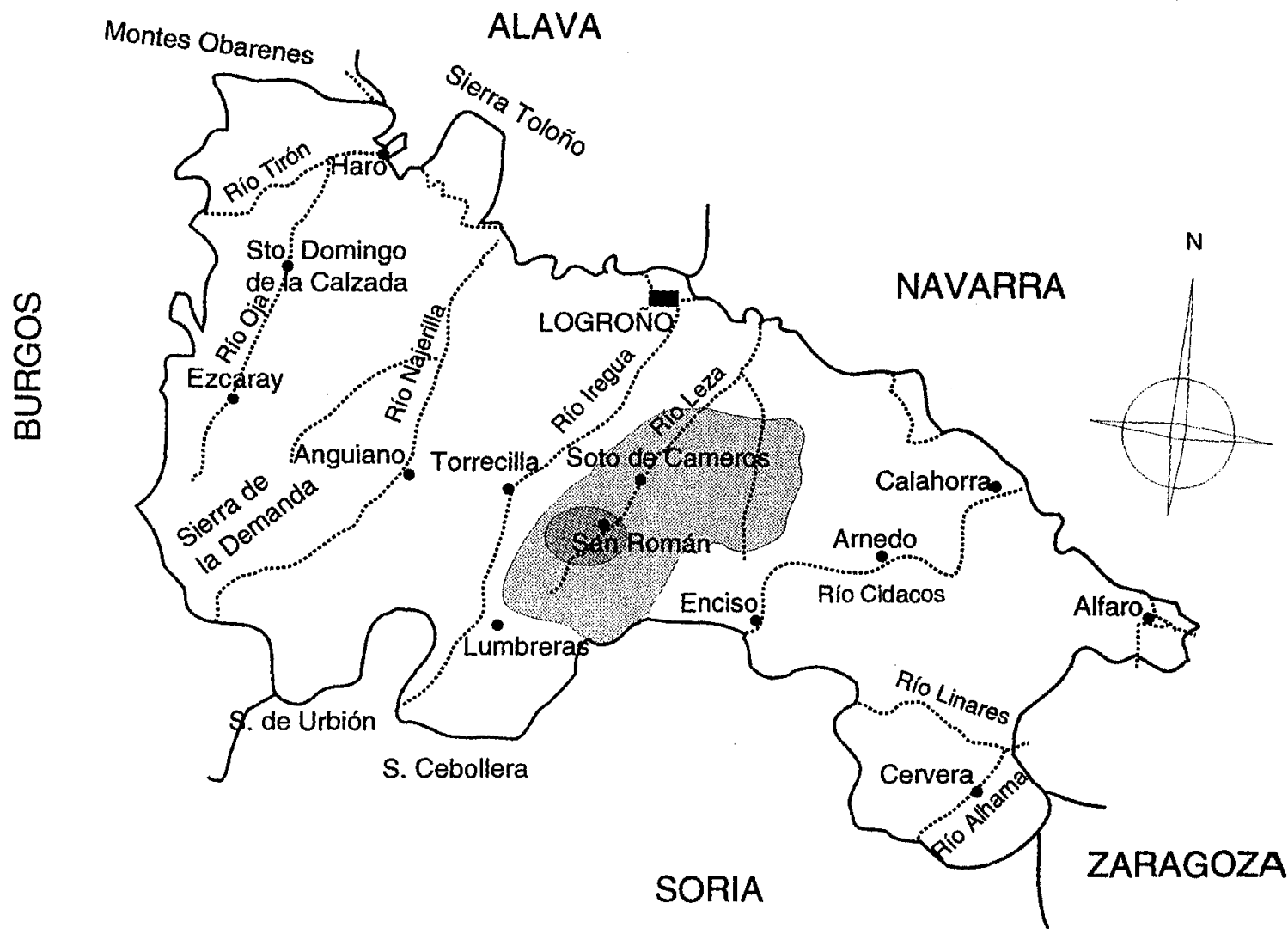

Localización de las parcelas muestreadas

Figura 1. Área de estudio

altitudes moderadas, comprendidas entre los 600 y $1700 \mathrm{~m}$ s.n.m. La práctica totalidad del área de estudio está ocupada por materiales mesozoicos, fundamentalmente del Cretácico, con dominio de cuarzoarenitas, arcillas arenosas, areniscas, margas y algunas intercalaciones calizas, en la facies Purbeck-Weald. Estos materiales están deformados en pliegues amplios, con buzamientos suaves. El sinclinal del Jubera es el accidente más importante (ITGE, 1990).

El relieve se caracteriza por una línea de cumbres muy suave, de formas pesadas y vertientes con desniveles generalmente moderados, si bien la red fluvial aparece encajada varios cientos de metros por debajo de las divisorias principales, por lo que las laderas próximas a ríos y barrancos alcanzan pendientes pronunciadas. En cualquier caso, Camero Viejo, de cara al aprovechamiento agropecuario, ofrece el aspecto de una media montaña mediterránea con pequeñas depresiones abiertas, cimas muy laxas y suaves laderas. Sólo el $12,1 \%$ del área de estudio posee desniveles superiores al 40\%, mientras que el $81,2 \%$ tiene del 10 al 40\% (Lasanta, 1989). 
El clima se caracteriza por registrar precipitaciones entre 600 y $1000 \mathrm{~mm}$ anuales y temperaturas relativamente frescas (en torno a $10-11^{\circ} \mathrm{C}$ de media anual). El régimen de precipitaciones es claramente equinoccial, siendo el verano la estación más seca. La mayor parte de la sierra entra dentro de la serie de Quercus pyrenaica. Sin embargo, la deforestación intensa que tuvo lugar en el pasado ha recluído al bosque a los enclaves de más difícil acceso o de escasa utilización agropecuaria. Las manchas más extensas aparecen en el sector meridional, correspondiendo algunas de ellas a bosques de repoblación (Fernández Aldana et al., 1989). En la actualidad la presencia masiva de matorral es el rasgo más representativo de su vegetación.

En 1996 estaban censados 809 personas en los pueblos incluidos en el área de estudio lo que da idea de su baja densidad demográfica $\left(2,4 \mathrm{hab} / \mathrm{Km}^{2}\right)$. El espacio cultivado no alcanza las $100 \mathrm{Ha}$, localizándose las mayores extensiones en el fondo del valle del Jubera, aguas arriba de Robres, y en las inmediaciones de Soto. Los censos ganaderos muestran el declive socioeconómico de Camero Viejo, ya que en 1994 había sólo 3.865 vacas, 3.307 ovejas y 2.894 cabras; cuarenta años atrás las cabaña ganadera ascendía a 555 vacas, 17.379 ovejas y 12.575 cabras (Lasanta y García-Ruiz, 1994). Se observa, pues, un descenso del ovino-caprino y un ascenso del vacuno, lo que tiene importantes consecuencias en la sucesión vegetal. Basta señalar inicialmente, que el vacuno permite el incremento de las formaciones de matorral, mientras que el ovino mantiene mejor los pastos dominados por comunidades herbáceas.

\section{Métodos}

A partir de trabajos precedentes se dispone de información sobre el proceso de abandono del espacio agrícola en relación con factores físicos y humanos (Lasanta et al., 1989; Arnáez et al., 1990; Oserín, 1996 y Lasanta et al., 1996), así como de los grandes rasgos de la sucesión secundaria (García-Ruiz et al., 1985; Sobrón y Ortiz, 1989).

Para este trabajo se seleccionaron 12 campos, localizados en los municipios de San Román, Jalón y Muro. Se eligieron seis campos con colonización vegetal dominada por aliaga (Genista scorpius) y otros seis con predominio de jara (Cistus laurifolius), al ser las dos especies más representativas de los modelos de sucesión vegetal que se dan en el área de estudio (Sobrón y Ortiz, 1989). Los seis primeros campos presentan un cubrimiento de herbáceas del 90-95\%, quedando la aliaga recluída a los bordes de la parcela. Los seis campos últimos poseen mayor cubrimiento de matorral (Cistus laurifolius), especialmente el 7, 8 y 9, en los que la jara alcanza casi el $50 \%$ de la 
superficie del suelo. Todos los campos se dejaron de cultivar en los años sesenta, siendo desde entonces pastoreados; en concreto cuatro campos se pastan exclusivamente con vacuno y el resto con ovino y vacuno, y en menor medida caprino y yeguas. En la tabla 1 se anotan las principales características de cada parcela muestreada. El rango de altitud oscila entre $830 \mathrm{~m}$ y 1045 $\mathrm{m}$. Las pendientes son suaves y hay campos en todas las exposiciones, menos la $S$. no hay grandes diferencias en la textura del suelo, distribuyéndose las granulometrías de forma bastante equilibrada. Respecto a la fertilidad destaca el campo número 7 que posee valores de materia orgánica, fósforo y potasio muy superiores al resto de las parcelas. Lo más destacado a comentar es el sustrato mucho más básico de los seis primeros campos frente al más silíceo de los últimos. El pH y los porcentajes de carbonatos y caliza activa así lo demuestran.

En cada campo se realizaron tres muestreos con un cuadrado de $0,5 \mathrm{~m}$ de lado. Se tomó información de porcentaje de cobertura vegetal, de suelo desnudo y pedregosidad, así como la frecuencia con que aparecía cada especie. Los muestreos se realizaron en primavera (abril), verano (julio y agosto) y otoño temprano (septiembre).

Para calcular el potencial forrajero se utilizó el método del "Valor Pastoral" de Daget y Poissonet (1979) ${ }^{3}$, en el que se aplica un índice a los distintos inventarios de vegetación. Este índice varía entre 0 y 100\% calculándose mediante la fórmula:

$$
\sqrt{\mathrm{P}=0,2 \mathrm{x} \sum \text { IsxCs, donde: }}
$$

Is = Indice de calidad específica que se atribuye a cada taxón, oscilando su valor entre 0 y 5 . Cuando la planta es rechazada por el ganado el valor es 0 y cuando la calidad forrajera es máxima 5.

\section{Cs= Contribución específica en tanto por ciento.}

Para dar el valor de Is se tienen en cuenta variables como el valor nutritivo, la apetecibilidad, el sabor, la asimilabilidad, la digestibilidad, etc. En este trabajo se han considerado los Is tanto por estaciones naturales del año como de forma global para todo el año, diferenciando -además- entre ovino y vacuno, dadas sus distintas preferencias de alimentación (Ferrer et al., 1993). Los valores utilizados

3 En el informe: "Caracterización y análisis de los recursos pastorales y su aprovechamiento en campos abandonados de Camero Viejo" se estimó también la producción y se realizaron análisis químico-bromatológicos a partir del método utilizado por Ferrer et al., (1990). 


\section{Municipio}

Altitud (m.s.n.m.)

Pendiente

Exposición

$\%$ Arenas

\% Limo

$\%$ Arcilla

$\mathrm{PH}$

Salinidad (C.E. 1:5)

\% Materia orgánica

Fósforo Olsen (ppm)

Potasio (ppm)

\% Carbonatos

\% Caliza activa

Magnesio (meq/100g suelo)

Pastoreo

Fuente: Rodríguez y Errea (1997)
S. R. S. R. S.R. S.R. S.R. S.R. Jalón Jalón Jalón Muro Muro Muro $\begin{array}{lllllllllllll}940 & 915 & 860 & 860 & 840 & 830 & 970 & 910 & 950 & 1045 & 1040 & 1045\end{array}$

$\begin{array}{llllllllllll}4 & 7 & 10 & 7 & 7 & 0 & 6 & 7 & 10 & 10 & 5 & 4\end{array}$

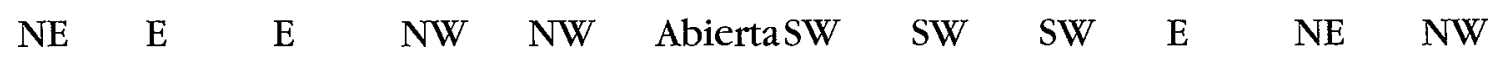

$\begin{array}{llllllllllll}20,7 & 27,0 & 29,6 & 37,2 & 39,3 & 38,8 & 30,2 & 42,7 & 39,5 & 41 & 38,9 & 25,9\end{array}$

$\begin{array}{llllllllllll}47,6 & 43,8 & 42,7 & 37,0 & 35,6 & 41,6 & 38,0 & 35,0 & 34,8 & 37,5 & 39,3 & 40,7\end{array}$

$\begin{array}{llllllllllll}31,7 & 29,1 & 27,7 & 25,7 & 25,1 & 19,6 & 31,9 & 22,4 & 25,6 & 21,5 & 21,7 & 33,4\end{array}$

$\begin{array}{llllllllllll}8,2 & 8,2 & 7,8 & 8,2 & 8,2 & 8,0 & 7,6 & 6,6 & 6,9 & 6,9 & 7,7 & 7,5\end{array}$

$\begin{array}{llllllllllll}0,17 & 0,16 & 0,18 & 0,11 & 0,10 & 0,11 & 0,20 & 0,09 & 0,07 & 0,06 & 0,19 & 0,22\end{array}$

$\begin{array}{lllllllllllll}6,08 & 7,14 & 8,92 & 4,69 & 4,25 & 5,11 & 11,75 & 5,71 & 7,04 & 6,56 & 5,25 & 6,98\end{array}$

$\begin{array}{llllllllllll}11,06 & 5,95 & 15,29 & 5,32 & 4,3 & 8,36 & 27,33 & 6,58 & 5,95 & 4,81 & 12,23 & 8,36\end{array}$

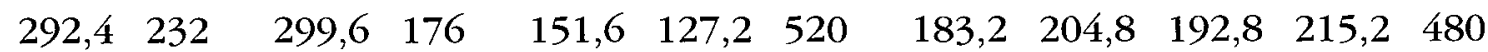

$\begin{array}{llllllllllll}29,04 & 36,94 & 20,04 & 11,14 & 13,16 & 8,61 & 9,09 & 1,67 & 1,4 & 3,38 & 3,08 & 1,63\end{array}$

$\begin{array}{llllll}6,9 & 4,3 & 7,5 & 3,9 & 3,4 & 2,9\end{array}$

$\begin{array}{llllllllllll}2,02 & 2,7 & 1,63 & 1,08 & 0,92 & 1,14 & 1,6 & 1,82 & 2,26 & 1,83 & 1,14 & 1,17\end{array}$

vacuno vacuno vacuno ovino/ ovino/ ovino/ ovino/ ovino/ vacuno ovino/ ovino/ ovino/

vacuno vacuno vacuno vacuno vacuno 
se han obtenido de la bibliografia (Daget y Poissonet, 1971; San Miguel, 1985, Ascaso, 1990 y Ferrer et al., 1993). Los Cs se han calculado a partir de los coeficientes de abundancia-dominancia de los inventarios fitosociológicos, transformados mediante las equivalencias ${ }^{4}$ dadas por otros estudios que utilizan el mismo método (Ascaso, 1990; Ferrer et al., 1993, Ascaso et al., 1996).

El valor pastoral es un índice relativo y adimensional que permite estimar la calidad del pasto, con valores que oscilan entre 0 y 100 , según la composición florística de los inventarios. Para conocer el potencial forrajero es necesario transformar el valor pastoral en Unidades Forrajeras (U.F.). Para esta transformación hemos utilizado la relación entre valor pastoral y UGM/ha/año establecida por Daget y Poissonet (1971) a partir de experiencias de campo con el ganado. La ecuación es:

$\mathrm{UGM} / \mathrm{ha} / \mathrm{año}=0,02^{*} \sqrt{ } \mathrm{P}$.

Si consideramos que 1 UGM consume al día 8,2 UF, dicha ecuación puede expresarse como: UF/ha/año $=60 * \sqrt{ }$ P. De esta forma se puede cuantificar aproximadamente la capacidad de carga que admite un espacio.

\section{Caracterización del espacio agrícola abandonado}

La máxima expansión del espacio cultivado se alcanzó en las últimas décadas del siglo XIX coincidiendo con el techo poblacional (García-Ruiz, 1988). Desde principios del siglo XX se asiste a un proceso de abandono que afectó al menos a 15.964 Ha (Tabla 2), según las estimaciones efectuadas a partir de cartografía realizada con fotografia aérea y la aplicación de un SIG. Hasta el año 1957 el abandono afectó al 31\% del área agrícola tradicional; en una segunda fase de recesión -entre 1957 y 1977- se abandonó otro 67\%. Desde 1977 se dejan de cultivar 409 Has más, quedando el área agrícola reducida a $99 \mathrm{Ha}$ (el 0,25\% de la superficie total y el 0,6\% del máximo histórico de superficie agrícola) (Lasanta et al., 1996).

Tabla 2: Superficie cultivada y sin cultivar en campo viejo a principios del siglo XX

\begin{tabular}{lccccc} 
& Superficie total & \multicolumn{2}{c}{ Superficie sin cultivar } & \multicolumn{2}{c}{ Superficie cultivada } \\
& $(\mathrm{Ha})$ & $(\mathrm{Ha})$ & $(\%)$ & $(\mathrm{Ha})$ & $(\%)$ \\
Leza & 27588 & 15992 & 57.97 & 11596 & 42.03 \\
Jubera & 11628 & 7260 & 62.44 & 4368 & 57.56 \\
TOTAL & 39216 & 23252 & & 15964 &
\end{tabular}

Fuente: Lasanta et al., (1996)

$4+=0,5 \%, 1=5 \%, 2=17,5 \%, 3=37,5 \%, 4=62,5 \%$ y $5=87,5 \%$ 
Con anterioridad a 1957 se abandonaron los peores campos, los localizados en laderas más pendientes y más alejados de los pueblos. A partir de los años sesenta el abandono fue masivo, sin grandes discriminaciones espaciales, al tratarse del cese total de la actividad agrícola en Camero Viejo (Lasanta et al., 1989). Tras el abandono, los campos se integran en el espacio pastoral en diferente grado, según su potencial pastoral y según las características propias del sistema ganadero de Camero Viejo. En este sentido, conviene señalar que desde los años cincuenta se asiste a la sustitución del ovino por el vacuno de aptitud cárnica (Fig. 2).

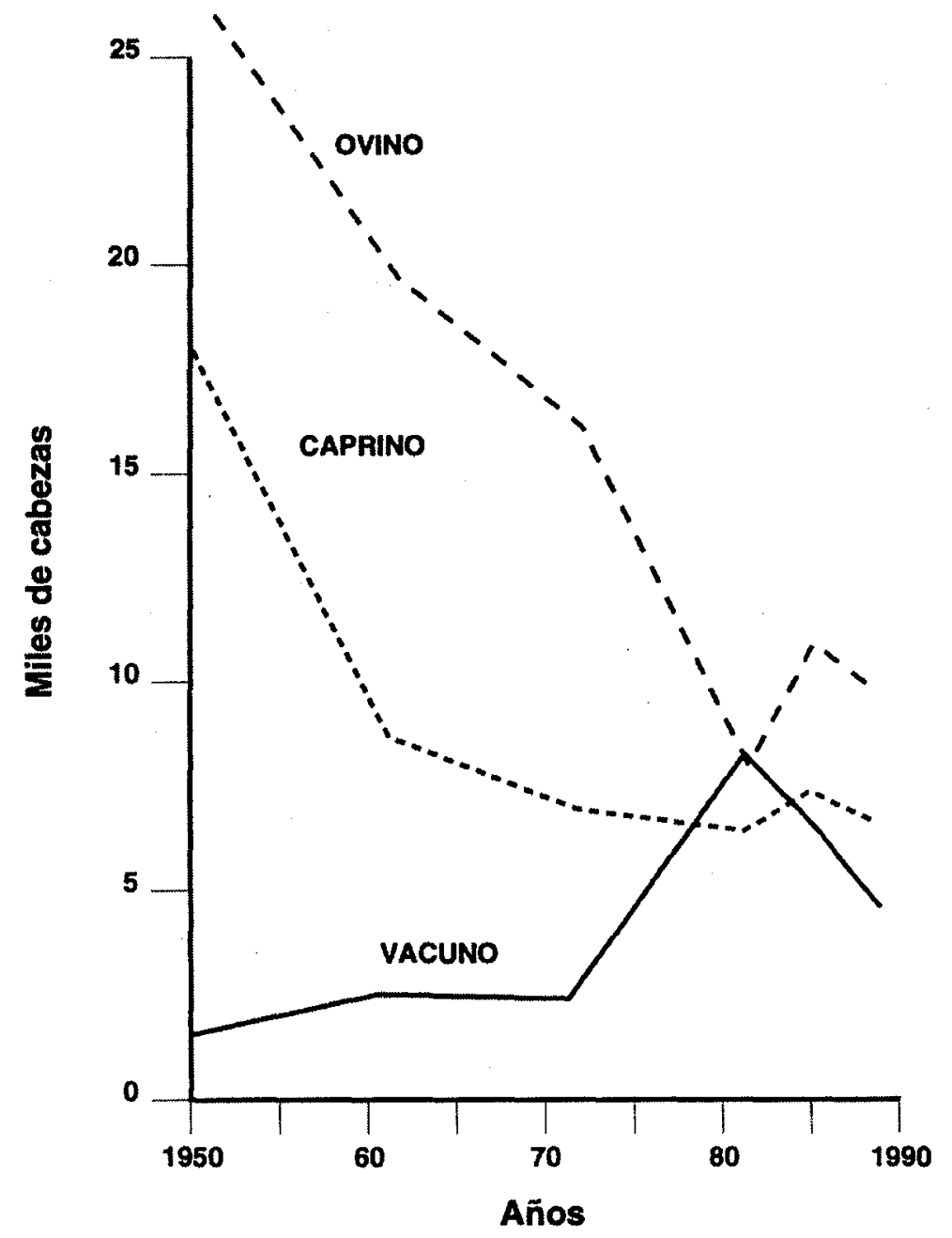

Figura 2. Evolución ganadera en Camero Viejo (1950 - 1990).

El incremento del vacuno se explica sobre todo por la ausencia de espacios a vigilar; la escasa superficie cultivada permite un pastoreo muy relajado sin apenas control sobre el ganado y, por tanto, con muy poca inversión de mano de obra. Simultáneamente al incremento de los censos de vacuno se produjo una sustitución de razas: la autóctona (Negra Avileña) fue sustituida por la 
Pardo-Alpina, la Charolesa y, en menor medida, por la Hereford, razas menos adaptadas a las condiciones ambientales de la sierra pero con mayores rendimientos en canal.

El pastoreo libre y el dominio de vacas poco adaptadas a las condiciones topográficas, implican el pastoreo frecuente de los mejores enclaves (fondos de valle y campos abandonados próximos a los pueblos) y un pastoreo ocasional o inexistente en el resto del territorio. Los campos abandonados más alejados, los situados en laderas más pendientes o los dejados de cultivar en las primeras fases de recesión no son visitados por el ganado (García-Ruiz y Lasanta, 1989). De esta forma se produce una utilización ganadera del espacio agrícola tradicional muy diferente. La fijación por las proximidades de los pueblos explica el mantenimiento de la productividad de los pastos en campos abandonados situados a poca distancia de los núcleos de población, mientras las áreas más alejadas evolucionan hacia un matorral denso que es cada vez menos frecuentado por el ganado. El propio sistema de manejo, sin forzar apenas el desplazamiento del ganado, contribuye a determinar la organización del paisaje, a controlar su productividad actual y futura, y a explicar en parte las pérdidas de suelo (Lasanta et al., 1996).

De cara al aprovechamiento ganadero resulta fundamental señalar los rasgos básicos del proceso de recolonización vegetal seguido por los campos abandonados del área de estudio. En pastizales mediterráneos la sucesión secundaria implica un cambio estructural y funcional en la vegetación, condicionado fundamentalmente por el marco ambiental abiótico y la interacción pasto-herbívoro. Las condiciones topográficas y edáficas de cada campo, la variabilidad climática y el comportamiento de los herbívoros son factores que condicionan la organización espacial, la variación de la composición florística y la productividad de los pastos. Desde el momento en que un campo deja de cultivarse, el ritmo y las características de la sucesión vegetal dependen del uso que se haga del campo, de las condiciones de fertilidad y humedad edáfica y del tipo de litología. De manera muy general y esquemática el proceso sigue la siguiente evolución: Se inicia con la rápida colonización por parte de herbáceas oportunistas y pioneras, que son sustituidas posteriormente por especies más longevas, que crean las condiciones edáficas adecuadas para la progresión de especies leñosas (matorrales fundamentalmente), de crecimiento más lento e importante almacenamiento de reservas, y otras de pastizal que capitalizan la energía en forma de celulosa y lignina, embasteciendo el pasto (Gonzalez Rebollar et al., 1993).

En los campos más visitados por el ganado el proceso sucesional puede detenerse a la vez que aumenta la adición de materia orgánica al suelo. De todas las formas suele desarrollarse una orla externa de aliagas (Genista scorpius) en suelos calizos, o ejemplares aislados de jaras (Cistus laurifolius) dentro del campo 
en ambientes silíceos. También es frecuente observar ejemplares aislados de Rubus ulmifolius y diferentes especies del genero Rosa. Por el contrario, en los campos más alejados, generalmente de mayor edad de abandono y muy poco pastoreados, el matorral alcanza coberturas notables, superando el $80 \%$ de la superficie a los 20-25 años de abandono. Conviene también señalar que si el sustrato es algo silíceo o se encuentra algo descarbonatado (como ocurre en las parcelas muestreadas en Jalón de Cameros) domina la jara, dejando muy poco espacio a las herbáceas. Sobre sustratos más calizos, Genista scorpius cubre progresivamente el campo. A los 3-4 años de abandono aparecen los primeros ejemplares en los márgenes del campo, avanzando cada año hacia el interior en forma de orla. Si la sucesión no es interrumpida (fuegos, desbroces o pastoreo) a los 25-30 años cubre totalmente el campo, si bien debajo aparece una densa cubierta de herbáceas (Sobrón y Ortiz, 1989, Molinillo et al., 1997).

\section{Valoración de los recursos pastables en campos abandonados de Camero Viejo.}

La tabla 3 incluye las especies más frecuentes en el estrato herbáceo-arbustivo de las parcelas estudiadas. Las leguminosas (14 especies) y las Gramíneas (12 especies) son las familias mejor representadas, lo que resulta muy interesante por ser las que aportan mayor valor a un pastizal. Aplicando el índice de Jaccard para establecer la clasificación de Bray-Curtis no se han encontrado diferencias significativas en la composición entre los estratos herbáceos de campos dominados por jara y los dominados por aliaga, si bien en los primeros el cubrimiento es mayor (Rodríguez y Errea, 1997).

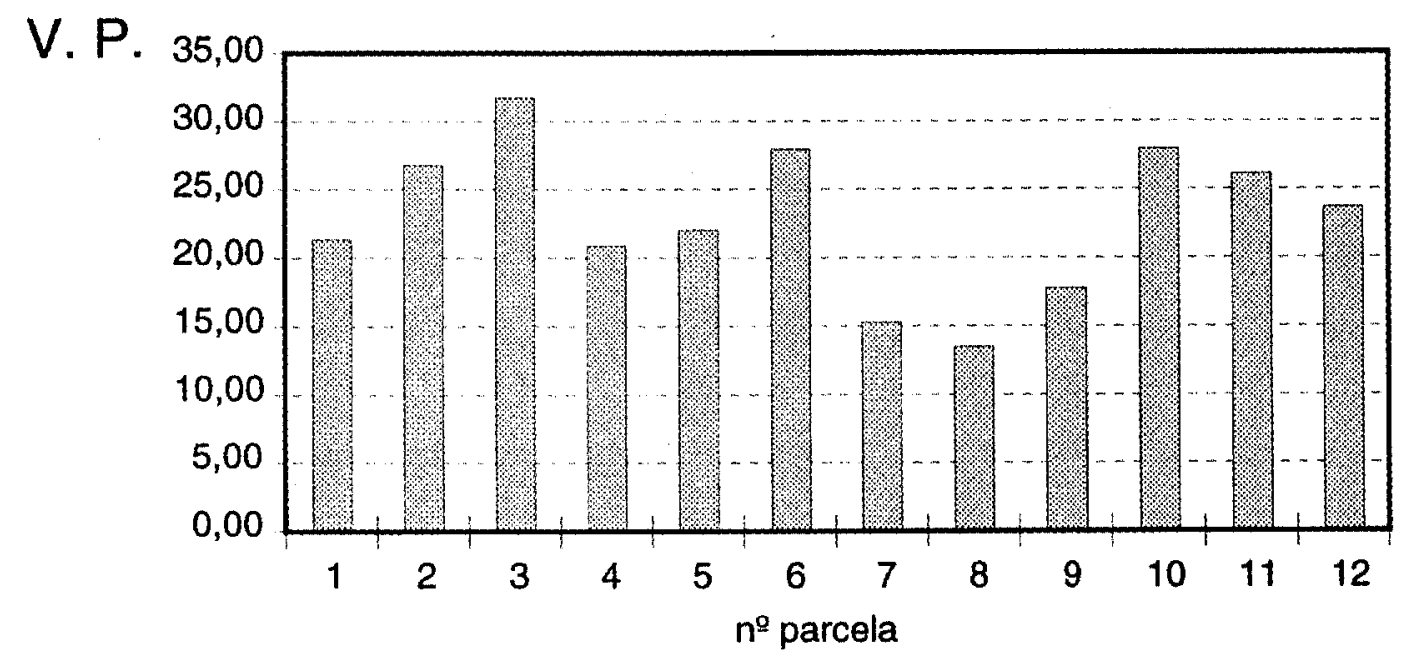

Figura 3. Valor Pastoral medio de las parcelas muestreadas. 
En la Fig. 3 se observa que no hay grandes diferencias en el Valor Pastoral medio, oscilando entre 13.2 en la parcela 8 y 31 en la parcela 3. En general, los pastos con predominio de Genista scorpius tienen un Valor Pastoral ligeramente superior (media de 24.8) a los de Cistus laurifolius (media de 20.4). Si el cálculo del Valor Pastoral se hace teniendo en cuenta las preferencias de consumo del ganado, todas las parcelas ofrecen mayor Valor Pastoral para el ovino que para el vacuno (media superior del 8.4\%), como consecuencia del mejor aprovechamiento que el ovino hace de flores, brotes tiernos y frutos de algunas leguminosas y de las especies espinosas abundantes en estos pastos (Ascaso et al., 1996).

La Fig. 4 muestra la evolución del Valor Pastoral desde abril a septiembre. Los valores más altos se registran en abril (valores comprendidos entre 41.3 y 20.5 para el ovino, y entre 38.6-16.5 para el vacuno), seguidos de los de septiembre (27.5-13.9 para el ovino y 26.3-14.1 para el vacuno) julio (22.4-7.8 para el ovino y 19.2-6.8 para el vacuno) y agosto (19.5-5.1 para el ovino y 16.9-3.9 para el vacuno). La primavera y el otoño constituyen, pues, las estaciones más productivas, coincidiendo con los máximos pluviométricos, mientras que en verano -especialmente en agosto- la productividad desciende por agostamiento de las hierbas. Este hecho pone de manifiesto el gran interés de los campos abandonados en primavera y otoño. A partir de mediados de julio, sin embargo, se produce un cambio en la dominancia de los taxones, apareciendo otros de menor Valor Pastoral. Las lluvias de finales de verano y principios de otoño favorecen el desarrollo de especies más palatables y un incremento de la cobertura. Por otro lado, de los resultados parece deducirse que en verano no existen grandes diferencias entre los campos de aliaga y los de jara, mientras que en primavera y otoño los primeros ofrecen mayor productividad.
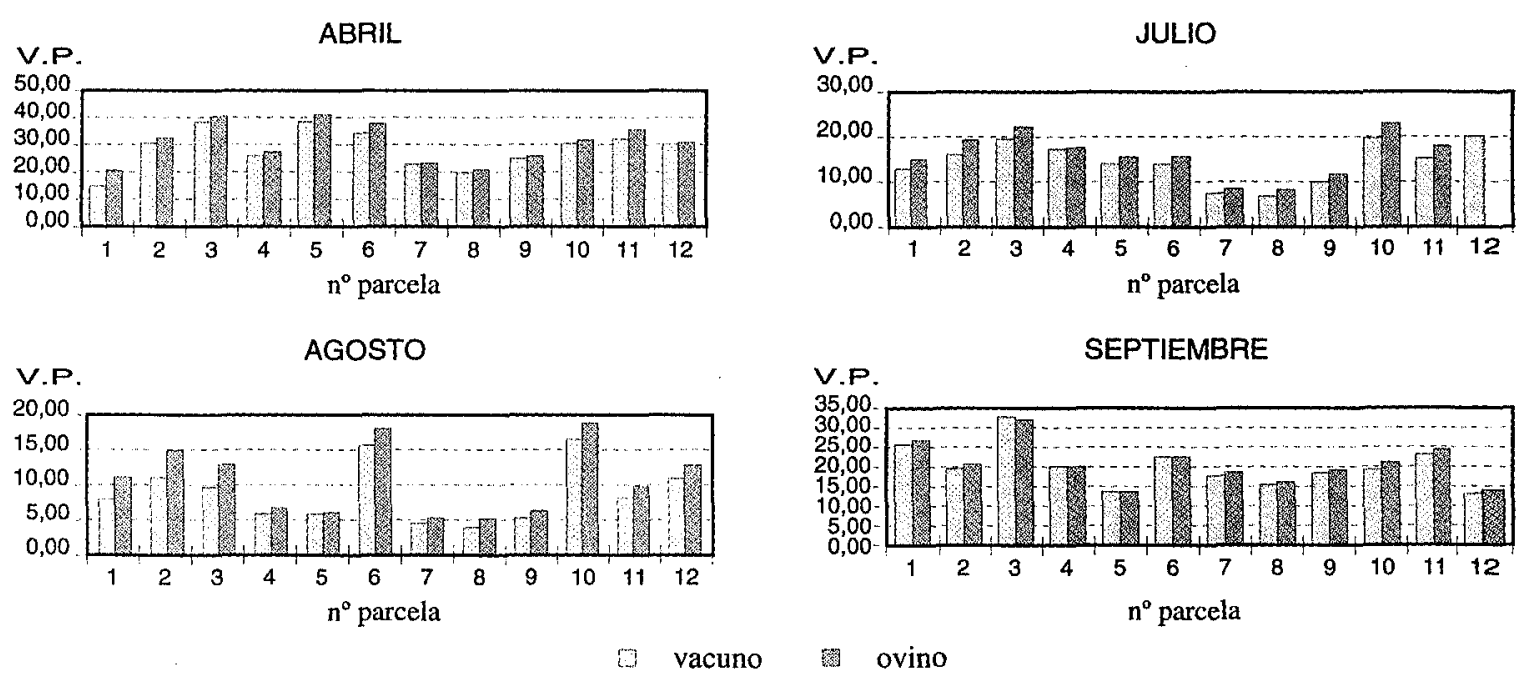

Figura 4. Valor Pastoral de las parcelas muestreadas (abril - septiembre). 
La tabla 4 anota la producción de las parcelas estudiadas en Unidades Forrajeras (U.F.) a partir del Valor Pastoral de cada campo. Conviene señalar que la producción debe ser ligeramente superior, al no incluirse la estación fría. Los valores medios oscilan entre 793 y $1861 \mathrm{UF} / \mathrm{ha} / \mathrm{año}$, presentando una variación menor que los obtenidos por Ferrer et al. (1993) en el Alto Maestrazgo y por Ascaso (1990) en el Prepirineo Aragonés. El campo menos productivo corresponde a una parcela cubierta por jara, que presenta bastante suelo desnudo en verano, mientras que en primavera y otoño crece Lolium multiflorum; hay que destacar también la aportación a finales de primavera de Lotus corniculatus y Ononis spinosa. En el campo más productivo dominan Medicago lupulina y Trifolium pratense en primavera y otoño. Se pone de manifiesto, al igual que en otros estudios de este tipo (Ascaso, 1990; Ferrer, 1988), que cuando la presencia de leguminosas es abundante (especialmente los generos Medicago y Trifolium) la producción y la calidad del pasto aumentan. También las especies del género Lolium son consideradas como buenas forrajeras. Sin embargo, en el caso de Camero Viejo su aportación es relativamente modesta, ya que aparecen con mucha frecuencia debajo de la jara, impidiendo ésta un buen aprovechamiento.

Tabla 3: Especies y familias determinadas en los muestreos realizados.

Familia

Cariofiláceas:

Cistaceas:

Compuestas:

Convolvuláceas:

Crucíferas:

Dipsacáceas:

Ericáceas:

Escrofulariáceas:

Gencianáceas:

Gramíneas:

\section{Especies}

Cerastium glomeratum

Cistus laurifolius, Helianthemun canum

Acbillea millefolium, Bellis perennis, Cicborium intybus, Filago pyramidata, Hieracium pilosella, Hypochoeris radicata, Santolina chamaecyparissus, Senecio jacobaea

\section{Convolvulus arvensis}

Capsella bursapastoris

Knautia purpurea, Scabiosa columbaria

Erica arborea

Veronica agrestis

\section{Centaurium tenuiflorum}

Arrbenatberum elatius, Bracbypodium ramosum, Bromus bordaceus, Cynosurus cristatus, Dactylis glomerata, Festuca rubra, Lolium multiflorum, Lolium perenne, Pbleum pratense, Poa annua, Triticum aestivum, Vulpia myuros

Labiadas: $\quad$ Lamium amplexicaule, Lavandula latifolia, Prunella laciniata, Teucrium chamaedrys, Thymus vulgaris 
Leguminosas: $\quad$ Dorycnium pentapbyllum, Genista scorpius, Lotus corniculatus, Medicago lupulina, Medicago orbicularis, Medicago polymorpha, Medicago sativa, Onobrychis bispanica, Ononis spinosa, Trifolium angustifolium, Trifolium campestre, Trifolium ocbroleucon, Trifolium pratense, Trifolium procumbens

Malváceas: $\quad$ Malva sylvestris

Plantagináceas: $\quad$ Plantago media

Polygaláceas: $\quad$ Polygala monspeliaca, Polygala nicaeensis

Ranunculáceas: Ranunculus bulbosus

Rosáceas: $\quad$ Agrimonia eupatoria, Geum sylvaticum, Rubus ulmifolius, Sanguisorba minor

Rubiáceas: $\quad$ Asperula arvensis, Asperula cynanchica, Galium mollugo, Galium verum, Sberardia arvensis

Umbelíferas: $\quad$ Daucus carota

Eryngium capestre

Tabla 4: Unidades forrajeras obtenidas a partir del valor pastoral. UF/ha/año en Camero Viejo

$\begin{array}{cccccccc}\text { Invent. } & \text { abril } & \text { julio } & \text { agosto } & \text { septiembre } & \text { Media } & \text { Med. Vacuno } & \text { Med. Ovino } \\ 1 & 1216,8 & 1287,6 & 844,8 & 1779,6 & 1282 & 967 & 1112 \\ 2 & 1862,4 & 1674,0 & 1150,8 & 1591,2 & 1570 & 1180 & 1338 \\ 3 & 2374,8 & 1876,8 & 1022,4 & 2169,6 & 1861 & 1516 & 1637 \\ 4 & 1358,4 & 1486,8 & 568,8 & 1534,8 & 1237 & 1036 & 1086 \\ 5 & 2149,2 & 1442,4 & 537,6 & 1114,8 & 1311 & 1092 & 1159 \\ 6 & 2124,0 & 1371,6 & 1464,0 & 1688,4 & 1662 & 1322 & 1435 \\ 7 & 1318,2 & 712,2 & 456,6 & 1194,0 & 920 & 808 & 867 \\ 8 & 1140,6 & 631,2 & 386,4 & 1014,6 & 793 & 705 & 763 \\ 9 & 1494,0 & 954,9 & 471,6 & 1215 & 1033 & 916 & 964 \\ 10 & 1777,8 & 1915,5 & 1555,5 & 1366,8 & 1653 & 1293 & 1443 \\ 11 & 1970,4 & 1576,8 & 811,2 & 1792,8 & 1538 & 1219 & 1359 \\ 12 & 1708,8 & 1885,2 & 1076,4 & 901,2 & 1393 & 1129 & 1222\end{array}$

Considerando que 1 UGM (1 vaca, equivalente a 8 ovejas) consume como media 8.2 UF/día se ha estimado la carga ganadera que admiten los campos abandonados muestreados. Como media sería de 0,45 UGM/ha/año, oscilando entre $0,3 \mathrm{UGM} / \mathrm{ha} /$ año en el de menor valor y $0,6 \mathrm{UGM} / \mathrm{ha} / \mathrm{año}$ en el de mayor producción. La capacidad de carga de los campos abandonados de Camero Viejo resulta ligeramente superior a la obtenida por Ascaso et al., (1996) en 
bancales abandonados del Maestrazgo, que la cifran en 0,25 UGM/ha/año. Si la estimación se hace diferenciando entre ovino y vacuno tendríamos que se podrían alimentar como media 3,2 ovejas/ha/año ó 0,37 vacas/ha/año. Como es lógico, al ser una relación lineal la que se establece entre el Valor Pastoral y las U.F., estas cargas aumentan en primavera y otoño y descienden en verano (agostamiento de la hierba) e invierno (parón del ciclo vegetativo).

Resulta difícil extrapolar estos resultados a la totalidad de Camero Viejo, dada la gran diversidad de microambientes vegetales que aparecen en campos abandonados. La cartografía de detalle (1: 5.000) realizada por encargo del Gobierno de La Rioja da una idea de la complejidad de estado sucesional en que se encuentran los campos abandonados, desde algunos en los que apenas hay matorral y todo el cubrimiento corresponde a herbáceas a otros donde el matorral invade la totalidad del campo. No obstante, una estimación a la baja, considerando la media de la parcela menos productiva, permite evaluar la capacidad de carga en 4.800 UGM, valor ligeramente superior a las 4.640 UGM que pastan en la actualidad. Por otro lado, el aprovechamiento ocasional del bosque y de otras laderas de matorral permite considerar una capacidad teórica de carga algo superior. Si, en cambio, se fuerza la producción de los mejores campos (los de menor pendiente) con desbroce del matorral y pastoreo continuado para favorecer el desarrollo de especies muy apetecibles para el ganado (Montserrat, 1964), la carga admisible sería muy superior. En este sentido, conviene señalar que el 55\% de los campos abandonados se localizan en laderas de menos del $20 \%$ de desnivel, por lo que al desbroce del matorral no ocasionaría graves problemas erosivos (Lasanta et al., 1996). Si la productividad de estos campos se mejorase hasta ofertar la de la parcela muestreada de mayor Valor Pastoral, la capacidad de carga ascendería a 7.423 UGM. En este sentido, existen ya experiencias positivas de recuperación de campos abandonados con fines pastorales, lo que no sólo permite aumentar la capacidad de carga de los campos mejorados sino también de las áreas próximas. Los campos recuperados se convierten en áreas atractivas (o cebos) para el ganado, que utiliza también los recursos próximos más bastos. De esta forma se crea una estructura más compleja de pastoreo que sienta las bases para un aprovechamiento integral de los recursos y para una mejora de la calidad del pasto a largo plazo (Valdelvira y Balcells, 1986). La publicación reciente de Matute y Chávarri (1998) sobre los pastos de La Rioja incide en la misma dirección, señalando la necesidad de desbrozar laderas para incrementar la producción pascícola. No obstante, no hay que olvidar que en invierno, y quizás en verano, sería necesario un complemento de pienso en pesebre. 


\section{Conclusiones y discusión}

Desde mediados del siglo $\mathrm{XX}$, Camero Viejo experimenta profundas transformaciones en sus sistemas de gestión siguiendo una evolución iniciada décadas atrás (Calvo Palacios, 1977; Lasanta y García-Ruiz, 1994). La tradicional ganadería trashumante de ovino y el cultivo cerealista del espacio agrícola han sido sustituidos por el vacuno de aptitud cárnica, que aprovecha fundamentalmente los antiguos campos agrícolas. Estos constituyen en la actualidad la principal fuente de alimentación de la ganadería de Camero Viejo.

En este trabajo se ha evaluado la producción y la capacidad de carga de doce campos abandonados y pastados habitualmente por el ganado. Los resultados muestran una alta heterogeneidad en función del sustrato litológico y de la gestión mediante pastoreo tras el cese de cultivo, factores ambos que determinan la sucesión vegetal y la composición florística. No obstante, de los resultados se pueden extraer algunas conclusiones, aplicables al área de estudio, muy generales:

- Los campos sometidos a mayor intensidad de pastoreo, siempre y cuando no se supere la capacidad de carga, son los que alcanzan mayor "Valor Pastoral". El ganado controla el avance del matorral y favorece la expansión de especies más apetecibles y nutritivas (Montserrat, 1964). Por otro lado, si el pastoreo es muy frecuente el ganado puede aprovechar las plantas en el momento óptimo.

- Las parcelas colonizadas por Genista scorpius ofrecen una productividad ligeramente superior a las de Cistus laurifolius, lo que se debe más a la mayor densidad del estado herbáceo en las primeras parcelas, que a la diversidad florística, que es muy parecida en ambos modelos de sucesión vegetal.

- La productividad alcanza su máximo primario en primavera y el secundario en otoño, mientras que desciende en verano, especialmente en agosto.

- Posiblemente los campos abandonados, en su situación actual de colonización vegetal, permiten alimentar una carga ganadera algo superior a la existente. Por otro lado, la incorporación de otros campos, en buenas condiciones topográficas pero muy invadidos por el matorral, mediante desbroce elevaría la capacidad de carga en un $60 \%$ respecto a la que hay actualmente. No obstante, existe la limitación de la alimentación invernal, que sólo se puede garantizar mediante importación de piensos o por la salida del ganado hacia el exterior. El cultivo de prados en Camero Viejo tiene escasas posibilidades, dadas las bajas precipitaciones que se registran y las dificultades para establecer un sistema de riego. 
De lo expuesto en las páginas precedentes se deduce que los campos abandonados de Camero Viejo tienen un buen potencial para el desarrollo de la ganadería extensiva, si bien el actual modelo de explotación (razas poco rústicas, escasa vigilancia del ganado, utilización muy discriminada del territorio con concentración en los campos más próximos a los establos y abandono del resto del territorio) no es el más apropiado para aprovechar todos los recursos pastorales. En cualquier caso, en áreas marginales de montaña -como Camero Viejo- desde la actual perspectiva socioeconómica (García-Ruiz y Lasanta, 1989), la ganadería extensiva constituye un buen sistema de aprovechamiento del territorio, porque permite rentabilizar con escasa inversión en mano de obra y en infraestructura el principal recurso (los pastos) que tienen este tipo de montañas. Por otro lado, hay que tener en cuenta que la ganadería extensiva no sólo cumple una función económica, sino también ecológica y social (Zorita, 1990; Sierra, 1996). En el primer caso, favorece el desarrollo de un paisaje más diversificado y con menos maleza, donde se controla la expansión generalizada del matorral, disminuyendo el riesgo de fuegos y consecuentemente la incentivación de procesos erosivos. En el segundo caso, porque garantiza la presencia del hombre en áreas que de lo contrario quedarían totalmente despobladas, ajenas a la dinámica socieconómica, y soportando pérdidas -a veces irreparables- en el patrimonio natural y cultural.

\section{Agradecimientos}

Parte de este trabajo se ha realizado gracias a una Ayuda de Investigación del Instituto de Estudios Riojanos (Gobierno de La Rioja) para elaborar el informe: Caracterización y análisis de los recursos pastorales y su aprovechamiento en campos abandonados de Camero Viejo.

\section{Referencias Bibliográficas}

Arnáez, J., Lasanta, T., Ortigosa, L.M. y Ruiz Flaño, P. (1990): L’abandon de l'espace agricole dans la montagne subméditerranéenne en Espagne (Pyrénées centrales et Système Ibérique). Revue Géographique des Pyrénées et du Sud-Ouest, 61(2) 237-253.

AsCASO, J. (1990): Estudio fitocenológico y valoración de los recursos pastorales de las zonas forestadas y arbustivas del Prepirineo aragonés. Institución Fernando el Católico: 152 pp., Zaragoza.

Ascaso, J., Ferrer, C. y Maestro M. (1996): Valoración estacional y anual de los recursos pastables en el Maestrazgo de Castellón. Actas de la XXXVI R.C. SEEP: 161-165, Logroño. 
Calvo Palacios, J.L. (1977): Los Cameros. De región bomogénea a espacioplan. Instituto de Estudios Riojanos, 2 vols, Logroño.

Daget, Ph y Poissonet, J. (1971): Une méthode d'analyse phitologique de prairies. Critéres d'application. Annales d'Agronomie, 22: 5-41.

Daget, Ph.y Poissonet, J. (1979); Un procede d'estimation de la valeur pastorale de pâturages. Fourrages, 49: 31-40.

ERREA, M.P. (1995): Desarrollo de la ganadería extensiva en relación con la retirada de tierras en la Depresión Central del Ebro. Master o Science. Instituto Agronómico Mediterráneo de Zaragoza: 216 pp., Zaragoza.

FERnÁNDEZ Aldana, R., Lopo, L. y Rodríguez, R. (1989): Mapa forestal de La Rioja. Instituto de Estudios Riojanos, 72 pp. + 1 mapa a escala 1: 100.000, Logroño.

Fernández GonZÁlez, L. (1980): Estudio ecológico del medio físico y de la vegetación de la Sierra de Cameros, en orden al aprovechamiento y conservación de los pastizales y monte bajo de la zona. Berceo, 98: 79-137.

FERNÁNDEZ GonzÁlez, L. (1985): Estudio fitoecológico de los pastizales de la Sierra de Cameros. Zubía, 3: 119-149.

Ferrer, C. (1988): Los recursos pascícolas del Pirineo aragonés. Ponencia XXVIII R.C. SEEP: 23-65, Jaca.

Ferrer, C., Amella, A., Maestro, M. y Ocaña, M. (1990): Estudio ecológico-agronómico. En: Explotación de pastos en caseríos guipuzcoanos (A. Amella y C. Ferrer, Eds): 203-253, Navarra.

Ferrer, C., Maestro, M., Hamrouni, S. y OcaÑa, M. (1993): Valoración de pastos del Alt Maestrat (Castellón). Informe: 624 pp., Zaragoza.

Francis, C. (1990): Variaciones sucesionales y estacionales de vegetación en campos abandonados de la provincia de Murcia, España. Ecología, 4: 35-47.

García-RuIZ, J.M. (1988): La evolución de la agricultura de montaña y sus efectos sobre la dinámica del paisaje. Revista de Estudios Agrosociales, 146: 7-37.

GARCÍA-RUIZ, J.M. y LASANTA, T. (1989): La ganadería extensiva en áreas montañosas marginales: algunos problemas teóricos y prácticos. Anales del Instituto de Estudios Agropecuarios, 11: 77-93.

GARCía-RuIZ, J.M., LASANTA, T. Y SOBRón, I. (1985): Estudio comparado de la evolución geomorfológica en campos abandonados y áreas repobladas de la cuenca del Jubera. Informe. Comunidad Autónoma de La Rioja: 345 pp., Logroño.

Gonzalez Rebollar, J.L., Passera, C.B. y De la Cruz, R. (1993): La intensificación del pastoreo y consecuencias no deseadas de la PAC. Paralelo 37, 16: 141-145.

ITGE (1990): Mapa geológico de España, Munilla 242 (1: 50.000). Instituto Tecnológico Geominero de España, Madrid. 
LASANTA, T. (1989): Cartografía y reincorporación al sistema productivo de laderas de campos abandonados en el Sistema Ibérico: valles del Leza y Jubera. Instituto de Estudios Riojanos (Informe): 79 pp. + mapas, Logroño.

LAsanta, T. y García-Ruiz, J.M. (1994): Los Cameros. En: Geografía de La Rioja (J.M. García-Ruiz y J. Arnáez, Edts). Caja Rioja, Tomo III: 145-191, Logroño.

Lasanta, T., Arnáez, J., Ortigosa, L.M. y Oserín, M. (1996): Consecuencias geoecológicas del abandono agrícola en Camero Viejo (Sistema Ibérico). Zubía, monográfico, 8: 61-85.

Lasanta, T., Arnáez, J., Ruiz Flaño, P. y Ortigosa, L.M. (1989): Evolución superficial del espacio cultivado en Camero Viejo (Sistema Ibérico) y su relación con algunos factores geoecológicos. Estudios Geográficos, 197: 553-572.

LLORENTE, J.M. Y LUENGO, M.A. (1986): El abandono de tierras: significado y gestión de las etapas de sucesión secundaria. El ejemplo de los relieves paleozoicos del W castellano-leonés. Monografías de L'E.Q.U.I.P, 2: 105-114, Barcelona.

Matute, P.P. y Chávarri, J.B. (1998): Los pastos en La Rioja. Cuaderno de Campo, 7: 36-39.

Molinillo, M., García-Ruiz, J.M. y Lasanta, T. (1994): Sucesión vegetal y recursos pastorales en campos abandonados del Pirineo Central. VIII Coloquio de Geografía Rural: 236-241, Córdoba.

Molinillo, M., Lasanta, T., García-Ruiz, J.M. (1997): Managing mountainous degraded landscapes after farmland abandonment in the Central Spanish Pyrenees. Environmental Management, 21(4): 587-598.

Montserrat, P. (1964): Ecología del pasto. Publicaciones del Centro Pirenaico de Biología Experimental, 1: 1-22.

MonTSERrat, G. (1990): Estudio de la colonización vegetal de los campos abandonados del valle de Aísa (Jaca, Huesca). Informe contribución al Proyecto LUCDEME: Erosión y colonización vegetal en campos abandonados.

OSERÍN, M. (1996): Caracterización y evolución geomorfológica de bancales abandonados en Camero Viejo (Sistema Ibérico riojano). Memoria de Iniciación a la Investigación. Universidad de La Rioja: 128 pp. + anéxos, Logroño.

PÉrez CHACón, E. Y VABRE, J. (1986): Cartografía y análisis multifactorial: métodos complementarios para el estudio de las alteraciones del paisaje a partir del abandono agrícola. Monografías de L'E.Q.U.I.P, 2: 189-198.

SAN Miguel, A. (1985): Ecología, tipología, valoración y alternativas silvopastorales de los quejigares (Quercus faginea Lamk.) y rebollo (Quercus pyrenaica). Tesis Doctoral, INIA, 63.

SIERra, I. (1996): Los sistemas extensivos, las razas autóctonas y el medio natural. Ponencia de la XXXVI R.C. SEEP: 17-31. Logroño. 
SOBRón, J. y ORTIZ, F. (1989): Aspectos de la colonización vegetal en un área de montaña submediterránea: el valle del Jubera (Sistema Ibérico, La Rioja). Cuadernos de Investigación Geográfica, 15: 89-108.

VALdelvira, A. y BaLcells, E. (1986): La pardina de Esporret como unidad empresarial de las sierras prepirenaicas meridionales. Pirineos, 128: 79-118.

ZoRITA, E. (1990): Hacia una nueva estructura de la ganadería ovina en España, armonizando recursos alimenticios y objetivos medioambientales. OVIS, 11: 9-42.

ZuAZÚA, M.T. (1987): Estudio de la sucesión secundaria en campos de cultivo abandonados en las tierras altas de la provincia de León. Tesis Doctoral. Universidad de León: 164 pp., más apéndices.

ZuAZÚA, M.T., GARZón, E. y CALlejA, A. (1988): Relación de la composición botánica de los pastizales desarrollados en campos abandonados con los factores edáficos y pluviométricos. Anales de la Facultad de Veterinaria de León, 34: 89-106. 
